\title{
Endothelial tyrosine kinase receptor B prevents VE-cadherin cleavage and protects against atherosclerotic lesion development in ApoE-/- mice
}

\author{
Hong Jiang ${ }^{1}$, Shuhong Huang ${ }^{2}$, Xinyun $\mathrm{Li}^{1}$, Xian $\mathrm{Li}^{2}$, Shanying Huang ${ }^{1}$, Yun Zhang ${ }^{1}$ \\ and Zhe-Yu Chen ${ }^{2}$ \\ ${ }^{1}$ Key Laboratory of Cardiovascular Remodeling and Function Research, Chinese Ministry of Education and Chinese Ministry \\ of Health, Qilu Hospital, Shandong University, Jinan, Shandong, China \\ 2 Department of Neurobiology, Shandong Provincial Key Laboratory of Mental Disorders, School of Medicine, Shandong \\ University, Jinan, Shandong, China \\ Correspondence to: Zhe-Yu Chen, email: zheyuchen@sdu.edu.cn \\ Yun Zhang, email: zhangyun@sdu.edu.cn
}

Keywords: atherosclerosis, endothelial barrier dysfunction, brain-derived neurotrophic factor, tyrosine kinase receptor B, vascular endothelial cadherin, Pathology Section

Received: June 30, $2015 \quad$ Accepted: September 06, $2015 \quad$ Published: September 28, 2015

This is an open-access article distributed under the terms of the Creative Commons Attribution License, which permits unrestricted use, distribution, and reproduction in any medium, provided the original author and source are credited.

\section{ABSTRACT}

Tyrosine kinase receptor $B$ (TrkB) is a high-affinity receptor for brain-derived neurotrophic factor (BDNF). In addition to its nervous system functions, TrkB is also expressed in the aortic endothelium. However, the effects of endothelial TrkB signaling on atherosclerosis remained unknown. Immunofluorescence analysis revealed that TrkB expression is downregulated in the endothelium of atherosclerotic lesions from ApoE-/- mice compared with the atheroma-free aorta of WT mice. Endothelial TrkB knockdown led to increased lesion size, lipid deposition and inflammatory responses in the atherosclerotic lesions of the ApoE-/- mice compared with the control mice. Mechanistic studies showed that TrkB activation prevented VE-cadherin shedding by enhancing the interaction between vascular endothelial protein tyrosine phosphatase and VE-cadherin, maintaining VE-cadherin in a dephosphorylated state. Our data demonstrate that TrkB is an endothelial injury-response molecule in atherogenesis. Endothelial BDNF/TrkB signaling reduces VE-cadherin shedding and protects against atherosclerotic lesion development in ApoE-/- mice.

\section{INTRODUCTION}

Tyrosine kinase receptor B (TrkB) is a highaffinity receptor for brain-derived neurotrophic factor (BDNF). The BDNF/TrkB pathway plays critical roles in the survival, growth, and maintenance of central and peripheral neurons [1]. In addition to its nervous system functions, BDNF/TrkB pathway also functions in the cardiovascular system. The BDNF/TrkB pathway has been reported to protect the myocardium against ischemic injury [2]. Constitutive BDNF/TrkB signaling is required for normal cardiac contraction and relaxation [3]. We and other researchers have reported that low plasma levels of BDNF associated with increased risk of cardiovascular disease [4-7]. BDNF deficiency results in endothelial cell apoptosis, intraventricular wall hemorrhage, depressed cardiac contractility and early postnatal death in mice [8]. Mice with a disrupted TrkB gene lack a significant proportion of intramyocardial blood vessels and showed early postnatal death [9]. We recently reported that TrkB was prominently expressed in the endothelium of atherosclerotic lesions and maintained endothelial barrier integrity by regulating VE-cadherin expression [10]. However, the association between endothelial TrkB signaling and atherosclerosis has not been determined.

In this study, we compared TrkB expression in aortas with and without atherosclerotic lesions in mice and investigated atherosclerotic lesion size, lipid and macrophage components, proinflammatory markers expression in ApoE-/- mice after knocking 
Table 1: Plasmid lipid contents of ApoE-/- mice transfected with AAV9-control, AAV9-shTrkB fed HCD for 12 weeks.

\begin{tabular}{|l|l|l|l|}
\hline Lipid $(\mathbf{m o l} / \mathbf{L})$ & AAV9-control $(\mathbf{n}=\mathbf{1 0})$ & AAV9-shTrkB $(\mathbf{n}=\mathbf{1 0})$ & $\boldsymbol{P}$ \\
\hline Total cholesterol & $23.9 \pm 6.6$ & $23.6 \pm 5.7$ & NS \\
Triglyceride & $1.14 \pm 0.58$ & $1.23 \pm 0.67$ & NS \\
LDL & $2.43 \pm 0.42$ & $2.47 \pm 0.81$ & NS \\
HDL & $6.33 \pm 0.71$ & $6.26 \pm 0.84$ & NS \\
\hline
\end{tabular}

down endothelial TrkB, seeking to reveal the role and mechanism of endothelial TrkB signaling in the development of atherosclerotic lesions.

\section{RESULTS}

TrkB expression in the endothelium is downregulated in atherosclerotic lesions of ApoE/- mice compared with the atheroma-free aorta of WT mice.

We performed immunofluorescence staining to compare the expression of $\mathrm{TrkB}$ in $\mathrm{C} 57 \mathrm{BL} / 6$ mice (controls) and ApoE-/- mice fed with an atherogenic diet for 8 weeks. In agreement with previous results [10], TrkB expression was prominent in the aortic ECs of atherosclerotic lesions. Importantly, the relative fluorescent intensity analysis demonstrated that the TrkB expression observed in the endothelium was decreased by $35 \%$ in the atheroma of ApoE-/- mice compared with the atheroma-free aorta of WT mice (Figure 1A, 1B). Considering that atherosclerosis is an inflammatory disease, then we investigated the effects of proinflammatory factors on TrkB expression in HAECs by administration with or without TNF- $\alpha$, ox-LDL. Western blot analysis demonstrated that TrkB expressions in HAECs were significantly inhibited after TNF- $\alpha$ or oxLDL administration (Figure 1C), which suggested that inflammatory factors might downregulate endothelial TrkB levels in atherosclerotic lesions.

A

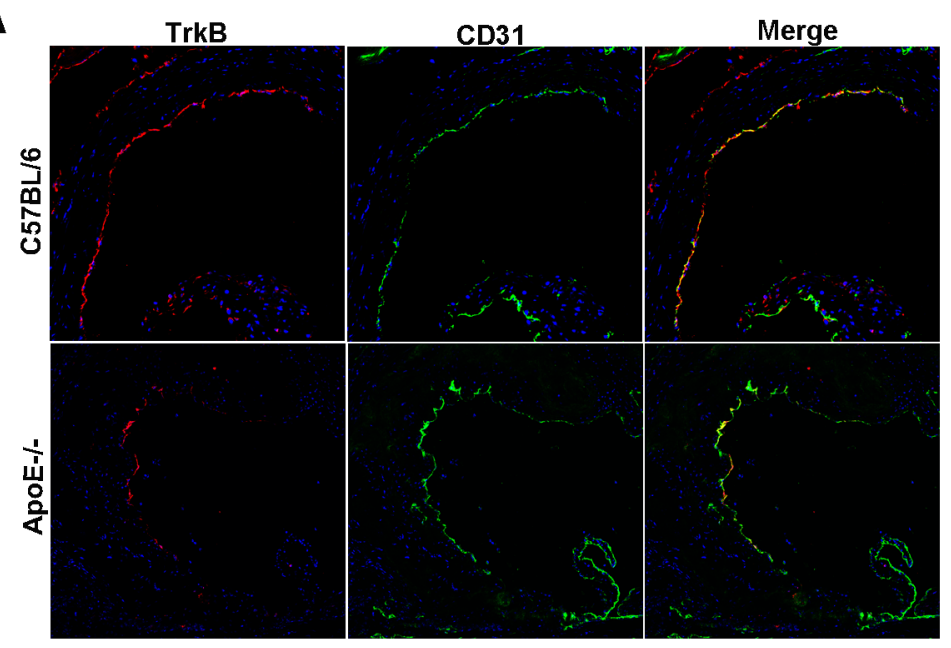

B

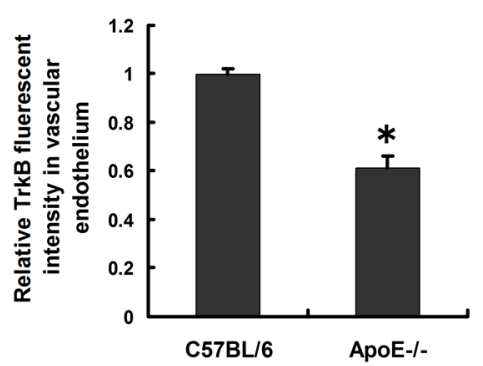

C

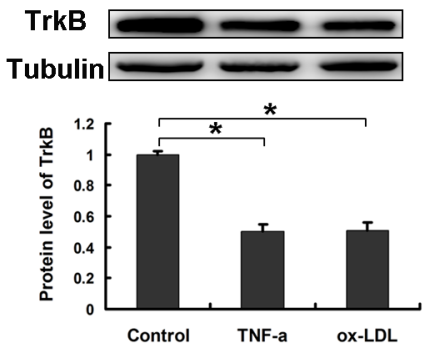

Figure 1: The expression of TrkB in endothelial cells was downregulated under atherosclerotic states. A., B. The expression of TrkB in atherosclerotic lesions of ApoE-/- mice was downregulated. A. Aortic root sections of WT $(n=5)$ or ApoE-/- $(n=5)$ mice fed a high-cholesterol diet for 8 weeks were subjected to immunofluorescence staining for TrkB and CD31. B. The relative fluorescent intensity of TrkB in the endothelium of aortic roots from WT and ApoE-/- mice ( $n=5$ sections per tissue, at least 3 analysis sites per slide). $* P<$ 0.05 vs. WT mice. C. TrkB expression in HAECs was decreased after stimulation with $50 \mathrm{ng} / \mathrm{mL}$ TNF- $\alpha$ or $40 \mu \mathrm{g} / \mathrm{ml}$ ox-LDL for 12 hours. 


\section{Endothelial TrkB confers atheroprotection in ApoE-/- mice}

Our previous study revealed that TrkB maintained endothelial barrier integrity [10]. Then the effects of endothelial TrkB knockdown on atherosclerosis were evaluated. ApoE-/- mice were systemically infected with Adeno-associated virus serotype-9 (AAV9) carrying a Zsgreen reporter gene (AAV9-control) or AAV9 carrying small hairpin RNA-TrkB (AAV9-shTrkB) via the tail vein and fed with an atherogenic diet for 12 weeks. After systemic infection, highly efficient expression of the reporter gene Zsgreen was observed in the endothelial layer of atherosclerotic lesions in ApoE-/- mice (Figure $2 \mathrm{~A})$. Immunofluorescence staining revealed a significant 91\% reduction of $\operatorname{TrkB}$ expression in the aorta of the ApoE-/- mice infected with AAV9-shTrkB compared with the control mice with AAV9-Control infection (Figure 2B), suggesting that the levels of TrkB in the aortic endothelial layer was efficiently knocked down.
The introduction of AAV9-shTrkB into apoE-/- mice significantly increased the lesion area in aortic trees compared with the introduction of AAV9-control (Figure 2C). Similar results were also found in the intimal area of aortic sinus cross-sections from the mice (Figure 2D, 2E). These mice displayed no change in food consumption and weight. The introduction of the two types of AAV9 did not significantly change the plasma concentration of lipids, including triglycerides and total, LDL, and high-density lipoprotein (Table 1). Our data revealed that endothelial TrkB confers atheroprotection in apoE-/- mice.

\section{Endothelial TrkB knockdown leads to increased lipid deposition, macrophage infiltration and inflammatory responses in the atherosclerotic lesions of ApoE-/- mice}

Then the lipid and macrophage deposition in the atherosclerotic lesions of ApoE-/- mice was evaluated. Lipid deposition in the lesion, as demonstrated by the
A

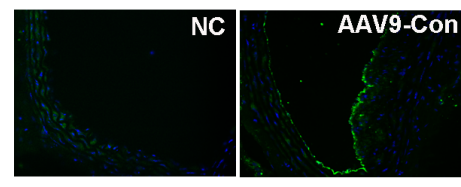

Green:Zsgreen Blue:DAPI
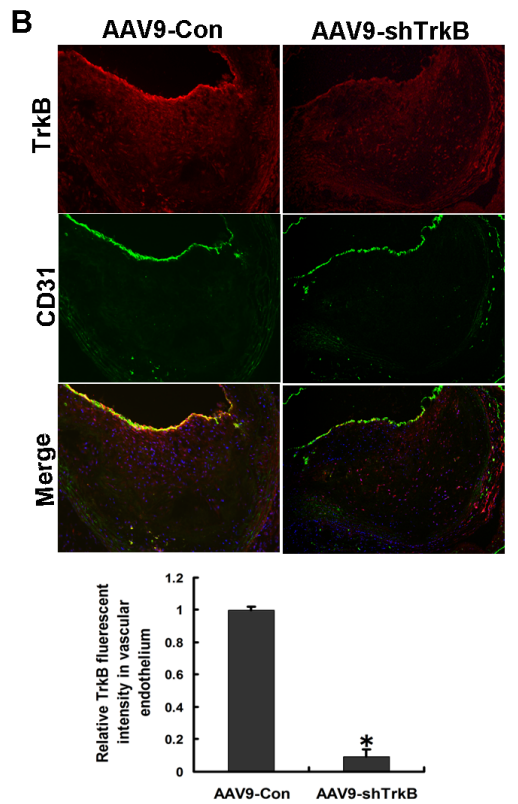

C

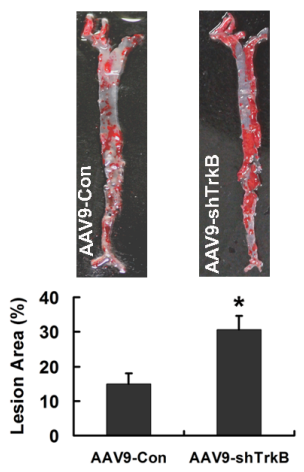

D
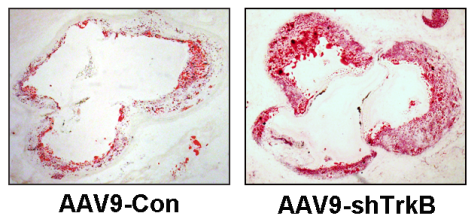

E

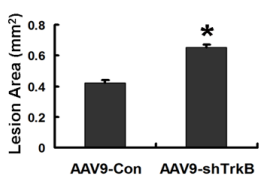

$\mathbf{F}$

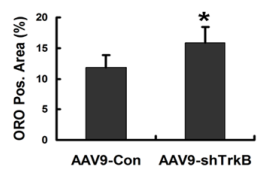

Figure 2: Endothelial TrkB confers atheroprotection in apoE-/- mice. Eight-week-old male ApoE-/- mice ( $n=10$ /group) were administered with AAV9-Con or AAV9-shTrkB via the tail vein, followed by feeding of a high-cholesterol diet for 12 weeks. A. The highly efficient expression of the reporter gene Zsgreen, carried by AAV9, in the aortic endothelial cells of the lesion. B. Double immunfluorescence staining for TrkB and CD31 and relative fluorescent intensity of TrkB in the endothelium of aortic roots from ApoE-/mice infected with AAV9-control or AAV9-shTrkB ( $n=5$ sections per tissue, at least 3 analysis sites per slide). C. En face staining of lesion areas with Oil Red $\mathrm{O}$ in the aorta. The data represent the percentage surface area of the aorta occupied by lesions in the ApoE-/- mice with or without TrkB knockdown. $* P<0.05$. D. Representative photographs of a cross section of the aortic sinus stained with Oil Red $O$ in the mice. E. The lesion sizes of the intimal area were measured using ImagePro-Plus. F. Quantitative computer-assisted image analysis of the lesions for lipid deposition. $* P<0.05$. 
Oil Red O-positive region, was significantly increased in ApoE-/- mice infected with AAV9-shTrkB (Figure 2F). The infiltration of macrophages into the vascular wall, as assessed by immunofluorescence with MOMA-2 antibody, was significantly increased with the AAV9-shTrkB (Figure 3A, 3B). Next, we investigated the inflammatory responses in the lesions. Real-time PCR showed that the mRNA levels of the proinflammatory markers, including nuclear factor- $\kappa \mathrm{B}$, intercellular adhesion molecule-1, vascular cell adhesion molecule-1, E-selectin, tumor necrosis factor- $\alpha$, and interleukin-6, in the aortas of ApoE-/- mice were significantly increased in the TrkB knockdown group compared with those in control group (Figure 3C). Our data revealed that TrkB protects aortas against lipoprotein leakage and monocyte extravasation and inflammatory responses during atherogenesis in ApoE-/- mice.

\section{BDNF prevented TNF- $\alpha$-induced-shedding of VE- cadherin in HAECs}

Our previous study revealed that TrkB promoted VE-cadherin synthesis in HAECs [10]. As expected, immunofluorescence staining showed that the VE- cadherin expression in atherosclerotic lesions was reduced by TrkB knockdown (Figure 4A). Importantly, by comparing the mRNA and protein levels of VE-cadherin after TrkB knockdown, we found that VE-cadherin protein was decreased by $79 \%$, whereas the VE-cadherin mRNA was only decreased by $43 \%$ (Figure $4 \mathrm{~B}, 4 \mathrm{C}$ ). These data suggested that TrkB might be involved in both the VEcadherin synthesis and cleavage.

It has been reported that an extracellular fragment of VE-cadherin can be cleaved and detected in medium [14]. Then we investigated whether BDNF-TrkB inhibited the shedding of VE-cadherin. As shown in Figure 4D, we found a $90 \mathrm{KD}$ shedding fragment of VE-cadherin in the medium consistent with previous study [15]. Importantly, the shedding fragment of VE-cadherin were significantly reduced in the medium of TNF- $\alpha$-stimulated HAECs by preincubation with BDNF, and the effects were blocked by TrkB siRNA, suggesting that BDNF prevented TNF- $\alpha$ induced shedding of VE-cadherin by TrkB activation.
A

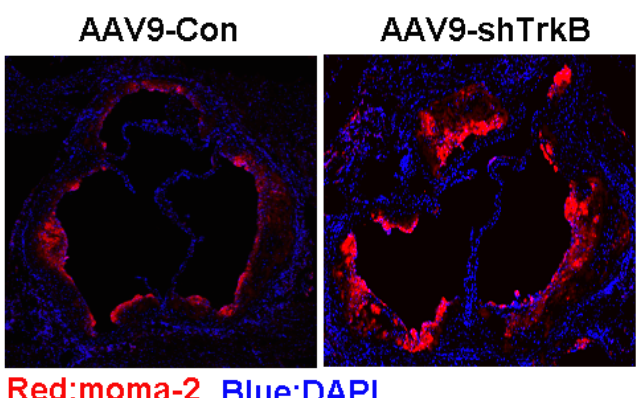

Red:moma-2 Blue:DAPI
B

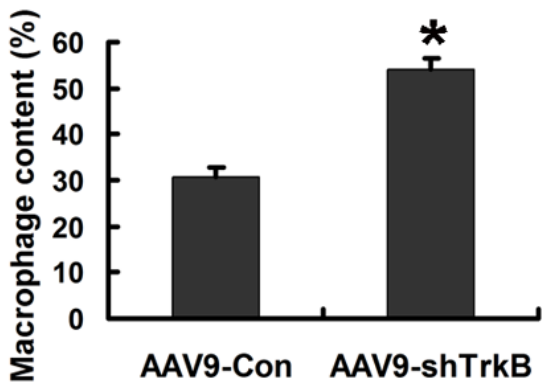

C
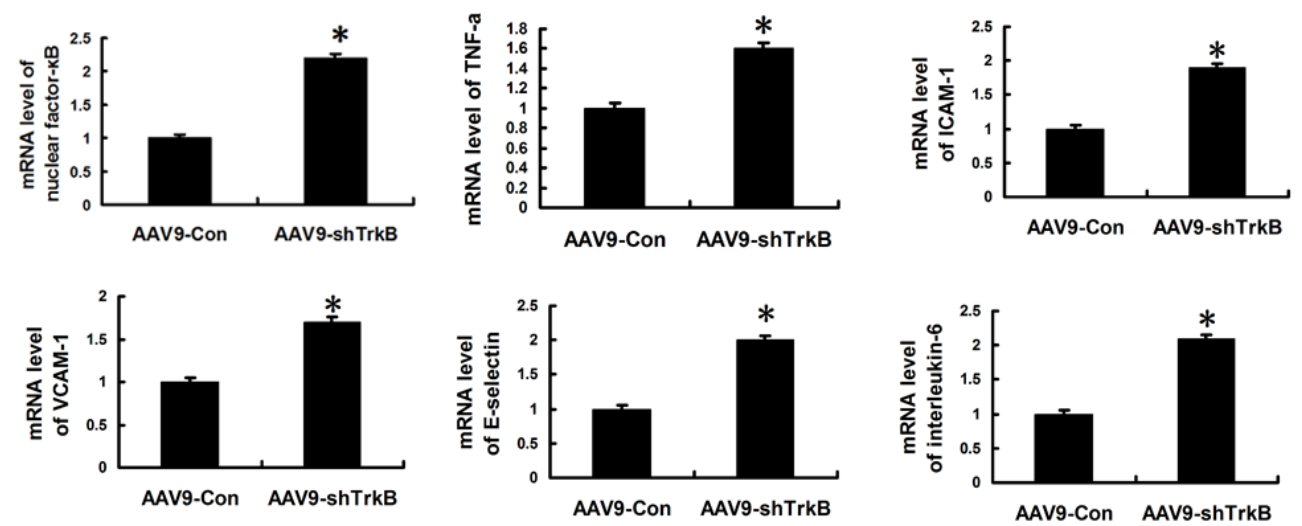

Figure 3: Increased macrophage infiltration and mRNA induction of proinflammatory markers in the atherosclerotic lesions of ApoE-/- mice by TrkB knockdown. A., B. Representative photographs of a cross section of the aortic sinus stained with moma-2. The macrophage contents in the lesions of the aortic sinus were measured. ${ }^{*} P<0.05$. C. Real-time PCR showed the mRNA levels of nuclear factor- $\kappa \mathrm{B}$, intercellular adhesion molecule-1, vascular cell adhesion molecule-1, E-selectin, tumor necrosis factor- $\alpha$, and interleukin- 6 in the aortas of the mice. $* P<0.05$. 
BDNF regulated tyrosine phosphorylation of $\mathrm{VE}$ cadherin and binding of VE-cadherin and VEPTP in HAECs

Tyrosine phosphorylation processes of VE-cadherin are required for the VE-cadherin cleavage [16]. Then, we investigated the effects of BDNF on the tyrosine phosphorylation levels of VE-cadherin. As reported by
Sidibé et al [16], we also found that TNF- $\alpha$ significantly induced the tyrosine phosphorylation of VE-cadherin. Importantly, BDNF reduced the tyrosine phosphorylation levels of VE-cadherin induced by TNF- $\alpha$, and the effects were blocked by TrkB siRNA (Figure 5A).

As a transmembrane binding partner of VEcadherin, vascular endothelial protein tyrosine phosphatase (VE-PTP) binds directly to VE-cadherin. The binding maintains VE-cadherin in a dephosphorylated state
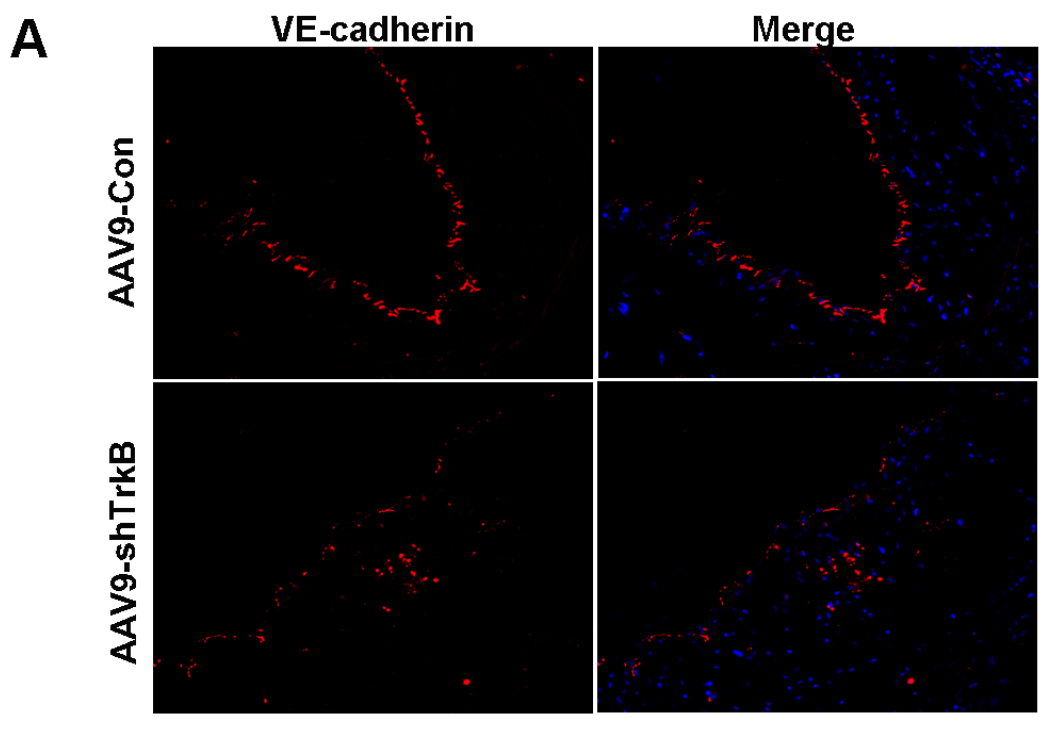

B
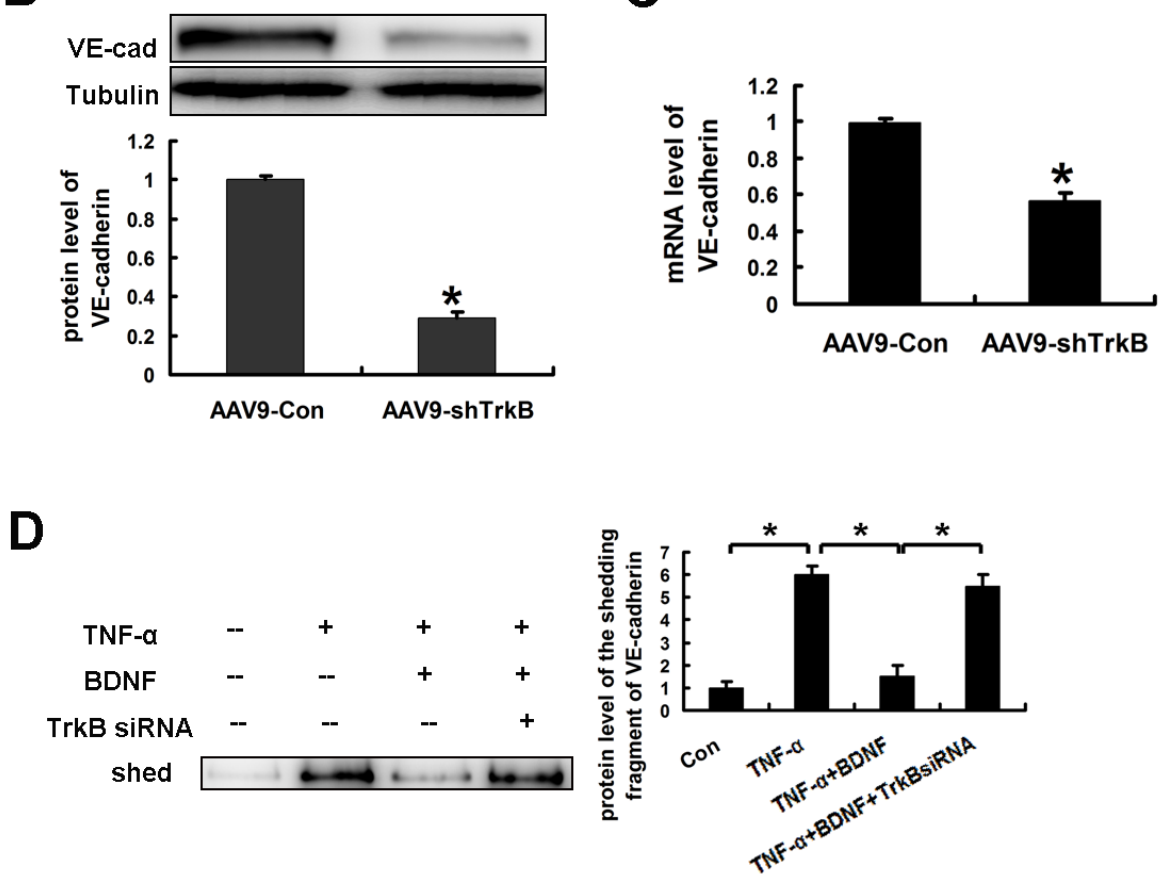

Figure 4: BDNF prevented TNF- $\alpha$ induced-shedding of VE-cadherin in HAECs. A. The expression of VE-cadherin in the endothelium in aortic lesions from the ApoE-/- mice with or without TrkB knockdown is shown by immunofluorescence staining. B. Realtime PCR and C. western blot analysis showed the mRNA and protein levels, respectively, of VE-cadherin in the aortas of the mice. D. The medium of HAECs treated as indicated was immunoprecipitated with anti-VE-cadherin antibody targeting its extracellular fragment and the immunoprecipitates were then examined for the shedding fragment using western blot analysis. 
[17]. Then, we evaluated whether BDNF promoted the binding of VE-PTP and VE-cadherin. The cells extracts of HAECs were immunoprecipitated with VE-cadherin antibody. As shown in Figure 5B, the levels of the immunoprecipitated VE-PTP were significantly higher in HAECs treated with BDNF, and the effects were blocked by TrkB siRNA. Similarly, the aortas of ApoE-/- mice with TrkB knockdown also displayed increased tyrosine phosphorylation of VE-cadherin and reduced binding of VE-PTP and VE-cadherin compared with control mice (Figure 5C, 5D).

A

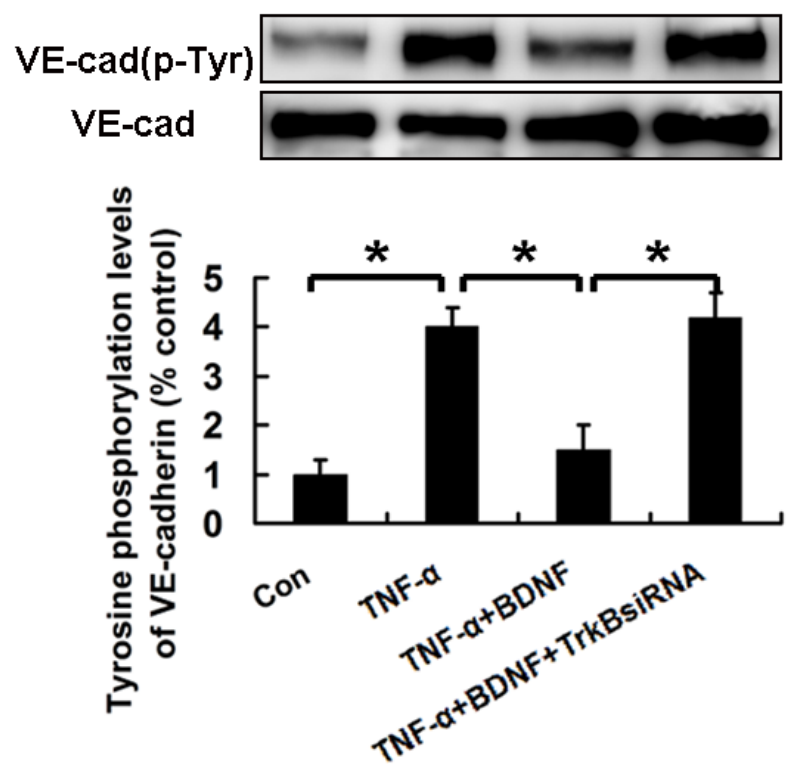

C

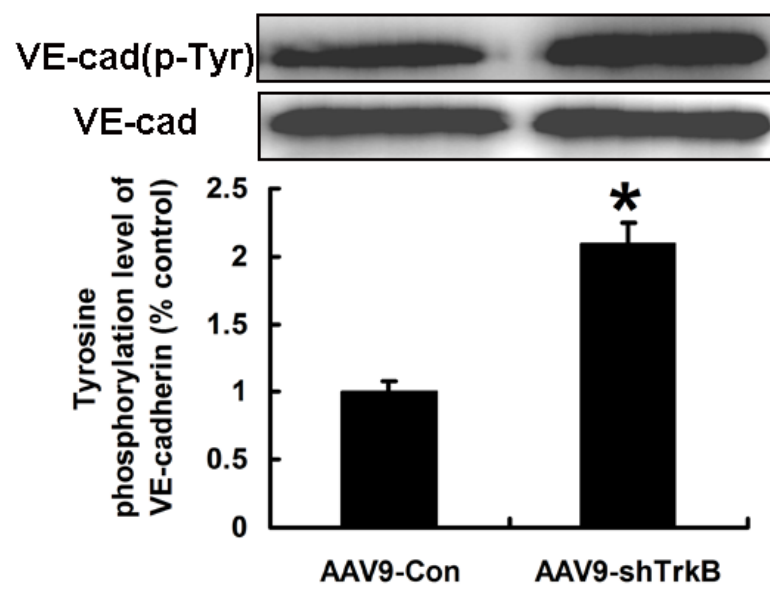

\section{DISCUSSION}

In this study, we investigated the role of endothelial TrkB signals in the development of atherosclerotic lesion in ApoE-/- mice. Our data showed that TrkB expression in the endothelium was downregulated in atherosclerotic lesions. Endothelial TrkB knockdown led to increased lesion size, lipid deposition and inflammatory responses in the atherosclerotic lesions of the ApoE-/- mice compared with the control mice. Finally, TrkB activation prevented the VE-cadherin shedding by maintaining the interaction between VE-PTP and VE-cadherin and reducing tyrosine

B

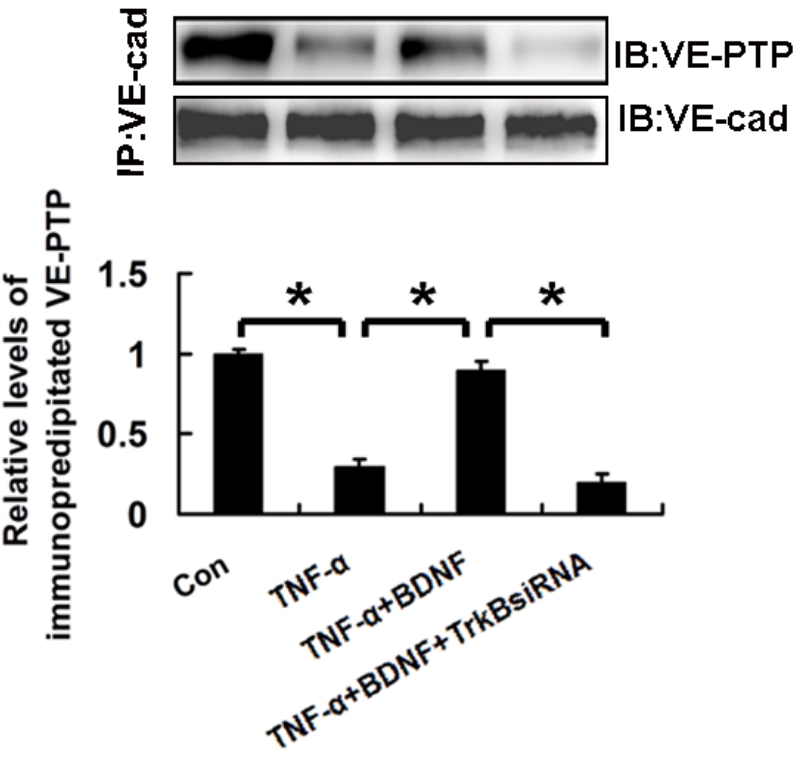

D

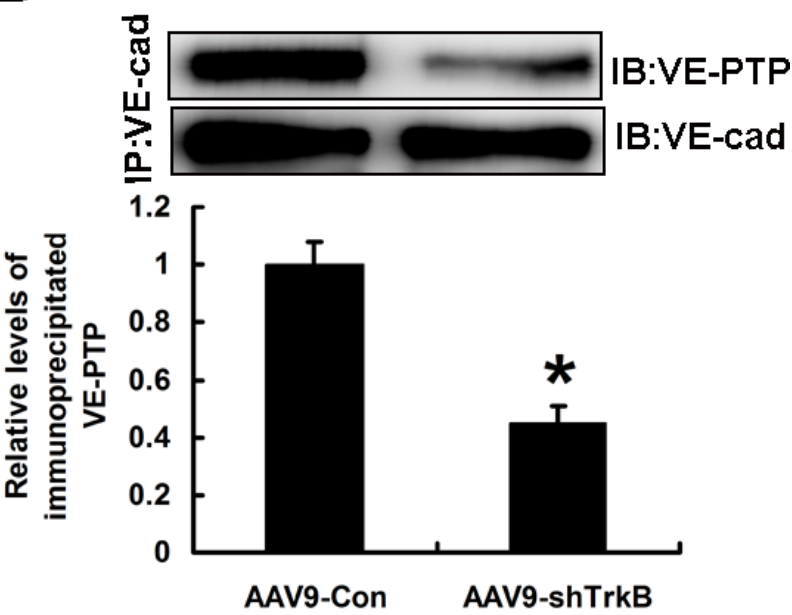

Figure 5: BDNF regulated tyrosine phosphorylation of VE-cadherin and binding of VE-cadherin and VE-PTP in HAECs. A., B. The cells extracts of HAECs treated as indicated were immunoprecipitated with VE-cadherin antibody and the immunoprecipitates were then examined using the A. anti-Phospho-Tyr antibody or B. anti-VE-PTP antibody. C., D. The extracts of aortas in ApoE-/- mice were immunoprecipitated with VE-cadherin antibody and the immunoprecipitates were then examined using the $\mathbf{C}$. antiPhospho-Tyr antibody or D. anti-VE-PTP antibody. ${ }^{*} P<0.05$. 
phosphorylation of VE-cadherin. Our data supported that endothelial TrkB signaling may be a novel therapeutic target for atherosclerosis.

TrkB is a high-affinity receptor for BDNF with wellestablished functions in the nervous system. However, TrkB is also expressed in the aortic endothelium. In this study, we found that TrkB expression in the endothelium to be downregulated in atherosclerotic lesions of ApoE/- mice compared with the atheroma-free aorta of WT mice. In vitro experiments we further demonstrated that the TrkB levels in HAECs were significantly decreased by proinflammatory factors. Our data suggest that TrkB is an endothelial injury-response molecule in atherogenesis and TrkB may participate in the pathologic process of atherosclerosis. Consistently, the decreased BDNF/TrkB levels were also found in the tissues of aged or patients with CAD-related complications. Decreased expression of BDNF in the endothelium was associated with hypertension [18]. In human umbilical vein endothelial cells the expression of BDNF was downregulated by laminar fluid shear stress [19]. In the hippocampus and temporal cortex, TrkB mRNA levels decreased with age [20]. The reason of reduced TrkB expression in injurytissues requires further investigations.

In this study, we revealed a novel role of endothelial TrkB in cardiovascular system as an atheroprotector. Our data demonstrated that endothelial BDNF-TrkB pathway deficiency led to accelerated development of atherosclerotic lesions in the aortic trees and aortic root of ApoE-/- mice. Similarly, as a growth factor signal, angiopoietin-1 has been reported to protect adult vasculature from leakage [21]. The administration of adenoviral Ang-2 to apoE-/- mice fed a Western diet significantly reduced the oxidized LDL and macrophage content of the plaques [22]. Therefore, our data revealed that the endothelial BDNF-TrkB pathway is a novel growth factor signal for protecting against atherosclerosis. Our previous study revealed that TrkB maintained endothelial barrier integrity by promoting VEcadherin expression [10]. In this study, our data further confirmed that $\mathrm{TrkB}$ protects aortas against lipoprotein leakage and monocyte extravasation and inflammatory responses during atherogenesis in ApoE-/- mice. The VEcadherin levels in the lesions of ApoE-/- mice with TrkB knockdown were also reduced. Therefore, the protective role of endothelia TrkB against atherosclerosis may be mainly mediated by maintaining VE-cadherin-dependent endothelial barrier function. However, our data appear to be in conflict with the results of Kraemer et al [23]. In Kraemer's paper, there was a $30 \%$ to $40 \%$ reduction in lesion size, witch caused by decreased smooth muscle cell accumulation, in TrkB $+/$ - mice compared with $\mathrm{TrkB}+/+$ mice. The reason may be that the expression of TrkB in the smooth muscle cells was not knockdowned in our study, because we don't observe the reporter gene expression of AAV9 vectors in smooth muscle cells of the aorta. Then the increased lesion size, lipid deposition and inflammatory responses are just from the endothelial TrkB knockdown in our study. Consistently, Kraemer also found macrophage immunoreactivity to be increased from $30 \%$ to $49 \%$ of the total lesion area in TrkB $+/$ - mice. Therefore, TrkB may stabilize atherosclerotic plaque via simultaneously maintaining endothelial barrier integrity and promoting smooth muscle cell activity.

In this study we found that BDNF/TrkB signaling prevented the TNF- $\alpha$ induced-VE-cadherin shedding. The elevated shedding fragment of VE-cadherin has been found to be associated with diabetic retinopathy and coronary atherosclerosis $[15,24]$. Thus the downregulation of TrkB in the endothelium in atherosclerotic lesions may be an underlying reason for elevated shedding fragments of VE-cadherin in patients with diabetic retinopathy or coronary atherosclerosis. We further elucidate the mechanism by which TrkB preventing the TNF- $\alpha$ inducedVE-cadherin shedding. Our data demonstrated that BDNF/ TrkB signaling involved in the tyrosine phosphorylation of VE-cadherin and the interaction between VE-PTP and VE-cadherin, leading to reduced endothelial cell-cell dissociation and hyperpermeability [25]. Therefore, our results provided a novel downstream molecular signaling of TrkB in protecting endothelial barrier integrity. How $\mathrm{BDNF} /$ TrkB regulates the interaction between VE-PTP and VE-cadherin is not clear and further research should be performed to elucidate these mechanisms.

Our data demonstrate that TrkB expression in the endothelium is downregulated in the atherosclerotic lesions of ApoE-/- mice. In addition to promoting the synthesis of VE-cadherin, endothelial BDNF/TrkB signaling also regulates the shedding of VE-cadherin and protects against atherosclerotic lesion development in ApoE-/- mice. Endothelial TrkB may be used as a novel therapeutic target in atherosclerosis.

\section{MATERIALS AND METHODS}

\section{Animal study protocol}

All procedures were performed in accordance with the Guide for the Care and Use of Laboratory Animals published by the U.S. National Institutes of Health (NIH Publication No.85-23, revised 1996) and were approved by the Ethics Committee of Shandong University. Eightweek-old male ApoE-/- mice received a single systemic administration of $2.1 \times 10^{11} \mathrm{vg} / \mathrm{g}$ body weight particles of adeno-associated virus serotype-9 (AAV9) with or without shRNA specifically targeting TrkB via the tail vein[11]. For TrkB knockdown, the shRNA sequences were 5'gaa cat caa gag cat cca ctt caa gag agt gga tgc tct tga tgt tct ttt tt-3' and 5'-aaa aaa gaa cat caa gag cat cca ctc tct tga agt gga tgc tct tga tgt tc-3'. To induce atherosclerotic 
lesion development, the ApoE-/- mice were fed a highcholesterol diet containing $42 \%$ fat and $0.2 \%$ cholesterol for 12 weeks. After overnight fasting, blood was collected via heart puncture for the measurement of plasma lipids. Then, the aortas were harvested for Oil Red O staining, immunofluorescence staining, and molecular biology analyses.

\section{Oil red $O$ staining}

Oil Red O staining was used to assess the size of the atherosclerotic lesion as previously described[12]. Briefly, the aortic trees and cross-sections of the aortic sinuses were fixed with 4\% paraformaldehyde. After washing with PBS, the aorta was opened longitudinally and pinned flat on a black surface. Then, the aortic trees and crosssections of the aortic sinuses were stained with Oil Red O (Sigma, USA). For quantification, the lesion size of the aorta and the intimal areas of the aortic sinuses were measured using the ImagePro-Plus software.

\section{Immunofluorescence analysis}

Immunofluorescence staining was used to show the expression of TrkB and VE-cadherin, and macrophage content of the lesions. Briefly, the cryosections of the aortic sinus were fixed with $4 \%$ paraformaldehyde. After washing with PBS and blocking with serum, the sections were incubated with anti-TrkB (sc-12), anti-CD31 (Abcam), anti-VE-cadherin (C-19; Santa Cruz), or antimouse macrophage (moma-2, AbD) antibodies. Then, the FITC or TRITC-conjugated secondary antibodies were used. Finally, the sections were observed using fluorescence microscopy.

\section{Western blot analysis}

Antibodies for anti-TrkB (sc-12), anti-VE-cadherin (C-19), and anti-vascular endothelial protein tyrosine phosphatase (sc-65228) were purchased from Santa Cruz. The preparation of the cell lysates, measurement of the protein concentrations, sodium dodecyl sulfatepolyacrylamide gel electrophoresis (SDS-PAGE), electrophoretic transfer, sequential incubation with primary and second antibodies and film development were performed as previously described[13].

\section{Real-time PCR}

Total RNA was isolated from the aortas of the mice. First cDNA was synthesized using AMV reverse transcriptase. PCR was performed using the following primers: forward, 5'-AGC AGG GAA ACA TCT ATA ACG-3' and reverse, 5'-CTT GAA CTT TGG GTT TAC
TGG-3' for mouse VE-cadherin; 5'-AGG CTT CTG GGC CTT ATG TG-3' and 5'-GTA TTC CTG GCG AGA GAA GCA-3' for NF- $\kappa$ B; 5'-GTG AAG GGA ATG GGT GTT3' and 5'-GGT CAC TGT CCC AGC ATC -3' for TNF- $\alpha$; 5'-TTC CAG AAA CCG CTA TGA-3' and 5'-GGT TGT CAC CAG CAT CAG-3' for IL-6; 5'-GGC ACC CAG CAG AAG TTG TT-3' and 5'-CTT GGT AGA GGT GAC TGA GG-3' for ICAM-1; 5'-TCT CTC AGG AAA TGC CAC CC-3' and 5'-GTG TGC TGC TAT TGG CTG TG3' for VCAM-1; 5'-TCC TCT GGA GAG TGG AGT GC-3' and 5'-ATG TGA AGC TTT GAC CCA CC-3' for E-selectin as well as 5' -TGT CTC CTG CGA CTT CAA CA-3' and 5'-GGT GGT CCA GGG TTT CTT ACT-3' for mouse GAPDH. The relative expression of the genes was obtained through the $2^{-\Delta \Delta \mathrm{Ct}}$ calculation.

\section{Cell culture}

Human aortic endothelial cells (HAEC) were purchased from ATCC (Manassas, VA). The HAECs were cultured in endothelial cell medium. All cells were cultured at $37^{\circ} \mathrm{C}$ with $5 \% \mathrm{CO} 2$. All assays were performed in triplicate.

\section{Statistical analysis}

The animal study data are presented as the mean \pm SEM. For comparisons between two groups, Student's $\mathrm{t}$ test was employed. All statistical tests were 2-tailed with $P<0.05$ set as the significance level, and they were performed using the SPSS 15.0 software (SPSS, Chicago, IL).

\section{ACKNOWLEDGMENTS AND GRANT SUPPORT}

This study was supported by the State Program of National Natural Science Foundation of China for Innovative Research Group (No 81321061), the National Natural Science Foundation of China (81200209, 81570228) and the Independent Innovation Foundation of Shandong University (2012TS138).

\section{CONFLICTS OF INTEREST}

All authors declare no conflicts of interest.

\section{REFERENCES}

1. Lewin GR, Barde YA. Physiology of the neurotrophins. Annual review of neuroscience 1996, 19:289-317.

2. Okada S, Yokoyama M, Toko H, Tateno K, Moriya J, Shimizu I, Nojima A, Ito T, Yoshida Y, Kobayashi Y, Katagiri H, Minamino T, Komuro I. Brain-derived 
neurotrophic factor protects against cardiac dysfunction after myocardial infarction via a central nervous systemmediated pathway. Arteriosclerosis, thrombosis, and vascular biology 2012, 32:1902-1909.

3. Feng N, Huke S, Zhu G, Tocchetti CG, Shi S, Aiba T, Kaludercic N, Hoover DB, Beck SE, Mankowski JL, Tomaselli GF, Bers DM, Kass DA et al. Constitutive $\mathrm{BDNF} /$ TrkB signaling is required for normal cardiac contraction and relaxation. Proceedings of the National Academy of Sciences of the United States of America 2015, 112:1880-1885.

4. Takashio S, Sugiyama S, Yamamuro M, Takahama H, Hayashi T, Sugano Y, Izumiya Y, Hokimoto S, Minamino N, Yasuda S, Anzai T, Ogawa H. Significance of Low Plasma Levels of Brain-Derived Neurotrophic Factor in Patients With Heart Failure. The American journal of cardiology 2015, 116:243-249.

5. Jiang H, Liu Y, Zhang Y, Chen ZY. Association of plasma brain-derived neurotrophic factor and cardiovascular risk factors and prognosis in angina pectoris. Biochemical and biophysical research communications 2011, 415:99-103.

6. Kaess BM, Preis SR, Lieb W, Beiser AS, Yang Q, Chen TC, Hengstenberg C, Erdmann J, Schunkert H, Seshadri S, Vasan RS, CardioGram, Assimes TL et al. Circulating brain-derived neurotrophic factor concentrations and the risk of cardiovascular disease in the community. Journal of the American Heart Association 2015, 4:e001544.

7. Fukushima A, Kinugawa S, Homma T, Masaki Y, Furihata T, Yokota T, Matsushima S, Takada S, Kadoguchi T, Oba K, Okita K, Tsutsui H. Serum brain-derived neurotropic factor level predicts adverse clinical outcomes in patients with heart failure. Journal of cardiac failure 2015, 21:300306.

8. Donovan MJ, Lin MI, Wiegn P, Ringstedt T, Kraemer R, Hahn R, Wang S, Ibanez CF, Rafii S, Hempstead BL. Brain derived neurotrophic factor is an endothelial cell survival factor required for intramyocardial vessel stabilization. Development 2000, 127:4531-4540.

9. Wagner N, Wagner KD, Theres H, Englert C, Schedl A, Scholz H. Coronary vessel development requires activation of the TrkB neurotrophin receptor by the Wilms' tumor transcription factor Wt1. Genes \& development 2005, 19:2631-2642.

10. Jiang H, Huang S, Li X, Li X, Zhang Y, Chen ZY. Tyrosine Kinase Receptor B Protects Against Coronary Artery Disease and Promotes Adult Vasculature Integrity by Regulating Ets1-Mediated VE-Cadherin Expression. Arteriosclerosis, thrombosis, and vascular biology 2015; 35:580-8.

11. Bostick B, Ghosh A, Yue Y, Long C, Duan D. Systemic AAV-9 transduction in mice is influenced by animal age but not by the route of administration. Gene therapy 2007, 14:1605-1609.

12. Guevara NV, Kim HS, Antonova EI, Chan L. The absence of p53 accelerates atherosclerosis by increasing cell proliferation in vivo. Nature medicine 1999, 5:335-339.

13. Sun $\mathrm{C}, \mathrm{Wu} \mathrm{MH}$, Guo M, Day ML, Lee ES, Yuan SY. ADAM15 regulates endothelial permeability and neutrophil migration via Src/ERK1/2 signalling. Cardiovascular research 2010, 87:348-355.

14. Herren B, Levkau B, Raines EW, Ross R: Cleavage of beta-catenin and plakoglobin and shedding of VE-cadherin during endothelial apoptosis. evidence for a role for caspases and metalloproteinases. Molecular biology of the cell 1998, 9:1589-1601.

15. Soeki T, Tamura Y, Shinohara H, Sakabe K, Onose Y, Fukuda N. Elevated concentration of soluble vascular endothelial cadherin is associated with coronary atherosclerosis. Circulation journal : official journal of the Japanese Circulation Society 2004, 68:1-5.

16. Sidibe A, Mannic T, Arboleas M, Subileau M, GulinoDebrac D, Bouillet L, Jan M, Vandhuick T, Le Loet X, Vittecoq O, Vilgrain I. Soluble VE-cadherin in rheumatoid arthritis patients correlates with disease activity: evidence for tumor necrosis factor alpha-induced VE-cadherin cleavage. Arthritis and rheumatism 2012, 64:77-87.

17. Nawroth R, Poell G, Ranft A, Kloep S, Samulowitz U, Fachinger G, Golding M, Shima DT, Deutsch U, Vestweber D. VE-PTP and VE-cadherin ectodomains interact to facilitate regulation of phosphorylation and cell contacts. The EMBO journal 2002, 21:4885-4895.

18. Prigent-Tessier A, Quirie A, Maguin-Gate K, Szostak J, Mossiat C, Nappey M, Devaux S, Marie C, Demougeot C. Physical training and hypertension have opposite effects on endothelial brain-derived neurotrophic factor expression. Cardiovascular research 2013, 100:374-382.

19. Nakahashi T, Fujimura H, Altar CA, Li J, Kambayashi J, Tandon NN, Sun B. Vascular endothelial cells synthesize and secrete brain-derived neurotrophic factor. FEBS letters 2000, 470:113-117.

20. Webster MJ, Herman MM, Kleinman JE, Shannon Weickert C. BDNF and trkB mRNA expression in the hippocampus and temporal cortex during the human lifespan. Gene expression patterns : GEP 2006, 6:941-951.

21. Thurston G, Rudge JS, Ioffe E, Zhou H, Ross L, Croll SD, Glazer N, Holash J, McDonald DM, Yancopoulos GD. Angiopoietin-1 protects the adult vasculature against plasma leakage. Nature medicine 2000, 6:460-463.

22. Ahmed A, Fujisawa T, Niu XL, Ahmad S, Al-Ani B, Chudasama K, Abbas A, Potluri R, Bhandari V, Findley CM, Lam GK, Huang J, Hewett PW et al. Angiopoietin-2 confers Atheroprotection in apoE-/- mice by inhibiting LDL oxidation via nitric oxide. Circulation research 2009, 104:1333-1336.

23. Kraemer R, Baker PJ, Kent KC, Ye Y, Han JJ, Tejada R, Silane M, Upmacis R, Deeb R, Chen Y, Levine DM, Hempstead B. Decreased neurotrophin TrkB receptor expression reduces lesion size in the apolipoprotein E-null mutant mouse. Circulation 2005, 112:3644-3653. 
24. Navaratna D, McGuire PG, Menicucci G, Das A. Proteolytic degradation of VE-cadherin alters the blood-retinal barrier in diabetes. Diabetes 2007, 56:2380-2387.

25. Angelini DJ, Hyun SW, Grigoryev DN, Garg P, Gong P, Singh IS, Passaniti A, Hasday JD, Goldblum SE. TNF-alpha increases tyrosine phosphorylation of vascular endothelial cadherin and opens the paracellular pathway through fyn activation in human lung endothelia. American journal of physiology Lung cellular and molecular physiology 2006, 291:L1232-1245. 